\title{
On sectoriality of degenerate elliptic operators
}

\author{
Tan Duc Do
}

\begin{abstract}
Let $c_{k l} \in W^{1, \infty}(\Omega, \mathbb{C})$ for all $k, l \in\{1, \ldots, d\}$ and $\Omega \subset \mathbb{R}^{d}$ be open with Lipschitz boundary. We consider the divergence form operator $A_{p}=-\sum_{k, l=1}^{d} \partial_{l}\left(c_{k l} \partial_{k}\right)$ in $L_{p}(\Omega)$ when the coefficient matrix satisfies $(C(x) \xi, \xi) \in \Sigma_{\theta}$ for all $x \in \Omega$ and $\xi \in \mathbb{C}^{d}$, where $\Sigma_{\theta}$ be the sector with vertex 0 and semi-angle $\theta$ in the complex plane. We show that a sectorial estimate hold for $A_{p}$ for all $p$ in a suitable range. We then apply these estimates to prove that the closure of $-A_{p}$ generates a holomorphic semigroup under further assumptions on the coefficients. The contractivity and consistency properties of these holomorphic semigroups are also considered.
\end{abstract}

November 2016

AMS Subject Classification: 35J25, 35K65, 47B44.

Keywords: Degenerate elliptic operator, sectorial operator, holomorphic semigroup, contraction semigroup, consistent semigroup.

\section{Home institution:}

Department of Mathematics

University of Auckland

Private bag 92019

Auckland 1142

New Zealand

Email: tan.do@auckland.ac.nz 


\section{Introduction}

In his book [Kat80] Kato showed that an $m$-sectorial operator in a Hilbert space generates a (quasi-)contraction holomorphic semigroup. One can generalise the notion of sectorial operators to $L_{p}$-spaces as follows (cf. [Gol85, Definition 1.5.8], [Kat80, Subsection V.3.10] and [CM05, Definition 1]).

Definition 1.1. Let $d \in \mathbb{N}, \Omega \subset \mathbb{R}^{d}$ be open and $p \in(1, \infty)$. Let $A_{p}$ be an operator in $L_{p}(\Omega)$. Then $A_{p}$ is said to be sectorial if there exists a $K>0$ such that

$$
\left|\operatorname{Im}\left(A_{p} u,|u|^{p-2} u \mathbb{1}_{[u \neq 0]}\right)\right| \leq K \operatorname{Re}\left(A_{p} u,|u|^{p-2} u \mathbb{1}_{[u \neq 0]}\right)
$$

for all $u \in D\left(A_{p}\right)$.

There are certain interests in showing that an operator is sectorial in this generalised sense. The significance of these estimates lies in the fact that they are useful in showing that the operators under consideration satisfy a necessary condition to generate holomorphic contraction semigroups. In particular the estimate (1) can be established for certain secondorder differential operators in divergence form. In the proof of [Paz83, Theorem 7.3.6], Pazy showed that (1) holds when the operator is strongly elliptic with symmetric realvalued $C^{1}$-coefficients, with an explicit constant $K$ which depends on the coefficients, the elliticity constant and $p$. Okazawa improved Pazy's result and showed that the estimate also holds for degenerate elliptic operators with symmetric real-valued $C^{1}$-coefficients, with $K=\frac{|p-2|}{2 \sqrt{p-1}}$ (cf. [Oka91]). Ouhabaz in Ouh05, Theorem 3.9] proved that (1) is true for generators of sub-Markovian semigroups. It is interesting to note that Ouh05, Theorem 3.9] gives the same constant $K$ in (11) as in [Oka91.

In this paper we will prove the sectorial estimate (11) for degenerate elliptic secondorder differential operators with bounded complex-valued coefficients. The results are generalisations of [Oka91]. In comparison to [Ouh05, Theorem 3.9], we note that the operators we consider here are in general no longer generators of sub-Markovian semigroups. We will then apply the estimate to show that degenerate elliptic operators with smooth enough coefficients generate contraction holomorphic semigroups.

In order to formulate the main theorem, we need to introduce some notation. Let $d \in \mathbb{N}, \Omega \subset \mathbb{R}^{d}$ be open with Lipschitz boundary and $\theta \in\left[0, \frac{\pi}{2}\right)$. Let $c_{k l} \in W^{1, \infty}(\Omega, \mathbb{C})$ for all $k, l \in\{1, \ldots, d\}$. Define $C=\left(c_{k l}\right)_{1 \leq k, l \leq d}$ and

$$
\Sigma_{\theta}=\left\{r e^{i \beta}: r \geq 0 \text { and }|\beta| \leq \theta\right\} .
$$

Assume that

$$
(C(x) \xi, \xi) \in \Sigma_{\theta}
$$

for all $x \in \Omega$ and $\xi \in \mathbb{C}^{d}$.

Let $p \in(1, \infty)$. Consider the operator $A_{p}$ in $L_{p}(\Omega)$ defined by

$$
A_{p} u=-\sum_{k, l=1}^{d} \partial_{l}\left(c_{k l} \partial_{k} u\right)
$$

on the domain

$$
D\left(A_{p}\right)=W^{2, p}(\Omega) \cap W_{0}^{1, p}(\Omega) .
$$

If $p=2$ then

$$
\left|\operatorname{Im}\left(A_{2} u, u\right)\right| \leq(\tan \theta) \operatorname{Re}\left(A_{2} u, u\right)
$$


for all $u \in D\left(A_{2}\right)$. This follows immediately from integration by parts. If $p \neq 2$ the situation is quite different. Write $C=R+i B$, where $R$ and $B$ are real matrices. Let $R_{a}$ and $B_{a}$ be the anti-symmetric parts of $R$ and $B$ respectively, that is, $R_{a}=\frac{R-R^{T}}{2}$ and $B_{a}=\frac{B-B^{T}}{2}$.

The main result of this paper is as follows.

Theorem 1.2. Suppose $\left|1-\frac{2}{p}\right|<\cos \theta$ and $B_{a}=0$. Then

$$
\left|\operatorname{Im}\left(A_{p} u,|u|^{p-2} u \mathbb{1}_{[u \neq 0]}\right)\right| \leq K \operatorname{Re}\left(A_{p} u,|u|^{p-2} u \mathbb{1}_{[u \neq 0]}\right)
$$

for all $u \in D\left(A_{p}\right)$, where

$$
K= \begin{cases}\tan \left(\frac{\pi}{2}-\phi+\theta\right) & \text { if } R_{a}=0 \\ \frac{\left(\frac{2}{\sin \phi}-1\right) \tan \theta+\cot \phi}{1-(\tan \theta) \cot \phi} & \text { if } R_{a} \neq 0\end{cases}
$$

and $\phi=\arccos \left|1-\frac{2}{p}\right|$.

Note that when the coefficient matrix $C$ consists of real entries and is symmetric, then one can choose $\theta=0$ and (5) gives

$$
K=\tan \left(\frac{\pi}{2}-\phi\right)=\cot \phi=\frac{|p-2|}{2 \sqrt{p-1}},
$$

which is the constant obtained by Okazawa in Oka91.

It is not difficult to see that $A_{p}$ is closable. Let $\overline{A_{p}}$ be the closure of $A_{p}$. Under the current conditions imposed on the coefficient matrix $C$ and the domain $\Omega$, we do not know whether $-\overline{A_{p}}$ is a generator of a $C_{0}$-semigroup. If $\Omega=\mathbb{R}^{d}$ and $C$ consists of twice differentiable entries, then we prove the following generation result for $-\overline{A_{p}}$ based on Theorem 1.2 .

Theorem 1.3. Let $\Omega=\mathbb{R}^{d}$. Suppose $\left|1-\frac{2}{p}\right|<\cos \theta$ and $B_{a}=0$. Suppose further that $c_{k l} \in W^{2, \infty}\left(\mathbb{R}^{d}\right)$ for all $k, l \in\{1, \ldots, d\}$. Set $\phi=\arccos \left|1-\frac{2}{p}\right|$. Then the closure $-\overline{A_{p}}$ generates a holomorphic semigroup on $L_{p}\left(\mathbb{R}^{d}\right)$ with angle $\psi$ given by

$$
\psi= \begin{cases}\phi-\theta, & \text { if } R_{a}=0 \\ \frac{\pi}{2}-\arctan \left(\frac{\left(\frac{2}{\sin \phi}-1\right) \tan \theta+\cot \phi}{1-(\tan \theta) \cot \phi}\right), & \text { if } R_{a} \neq 0\end{cases}
$$

Note that

$$
\psi_{1}:=\frac{\pi}{2}-\arctan \left(\frac{\left(\frac{2}{\sin \phi}-1\right) \tan \theta+\cot \phi}{1-(\tan \theta) \cot \phi}\right) \leq \phi-\theta
$$

since

$$
\tan \psi_{1}=\frac{1-(\tan \theta) \cot \phi}{\left(\frac{2}{\sin \phi}-1\right) \tan \theta+\cot \phi} \leq \frac{1-(\tan \theta) \cot \phi}{\tan \theta+\cot \phi}=\tan (\phi-\theta) .
$$

It is also interesting that in the case when $R_{a}=0$, Theorem 1.3 provides better angles of holomorphy compared with those of Stein's interpolations [Ouh05, Proposition 3.12] and [Ste70, Theorem 1]. In the one-dimensional case, these better angles were also obtained in [DE16, Corollary 1.3]. Other results about angles of holomorphy were considered in [Wei, Theorem 1], Epp89, Theorem 1.1], [Dav89, Theorem 1.4.2], [RS75, Theorem X.55], [LP95] and Ouh05, Theorems 3.12 and 3.13].

The holomorphic semigroup generated by $-\overline{A_{p}}$ in Theorem 1.3 also possesses nice contractivity and consistency properties. 
Theorem 1.4. Adopt the assumptions and notation as in Theorem 1.3. Let $S^{(p)}$ be the semigroup generated by $-\overline{A_{p}}$ and $S$ the semigroup generated by $-\overline{A_{2}}$. Then the following hold.

(i) $S^{(p)}$ is contractive on $\Sigma_{\gamma}$, where

$$
\gamma= \begin{cases}\psi & \text { if } R_{a}=0 \\ \psi \wedge \sup \left\{\beta \in\left[0, \frac{\pi}{2}\right):(\tan \theta) \tan \beta<\frac{1}{3}\right\} & \text { if } R_{a} \neq 0 .\end{cases}
$$

(ii) $S^{(p)}$ is consistent with $S$ on $\Sigma_{\psi}$.

The outline of subsequent sections are as follows. In Section 2 we provide some estimates on the coefficient matrix $C$. These estimates are used to prove Theorem 1.2 in Section 3 . Theorems 1.3 and 1.4 are proved in Section 4 .

\section{Estimates on coefficients}

Let $\Omega, \theta$ and $C$ be as in Section 1 . In this section we provide some preliminary estimates on the coefficient matrix $C$ for later use.

Define

$$
\operatorname{Re} C=\frac{C+C^{*}}{2} \text { and } \operatorname{Im} C=\frac{C-C^{*}}{2 i},
$$

where $C^{*}$ is the conjugate transpose of $C$. Then $(\operatorname{Re} C)(x)$ and $(\operatorname{Im} C)(x)$ are self-adjoint for all $x \in \Omega$ and

$$
C=\operatorname{Re} C+i \operatorname{Im} C .
$$

We will also decompose the coefficient matrix $C$ into

$$
C=R+i B
$$

where $R$ and $B$ are matrices with real entries. Write $R=R_{s}+R_{a}$, where $R_{s}=\frac{R+R^{T}}{2}$ is the symmetric part of $R$ and $R_{a}=\frac{R-R^{T}}{2}$ is the anti-symmetric part of $R$. Similarly $B=B_{s}+B_{a}$, where $B_{s}=\frac{B+B^{T}}{2}$ and $B_{a}=\frac{B-B^{T}}{2}$. It follows from (9) and (10) that

$$
\operatorname{Re} C=R_{s}+i B_{a} \text { and } \operatorname{Im} C=B_{s}-i R_{a} .
$$

Lemma 2.1. We have

$$
\left|\left(R_{s} \xi, \eta\right)\right| \leq \frac{1}{2}\left(\left(R_{s} \xi, \xi\right)+\left(R_{s} \eta, \eta\right)\right)
$$

for all $\xi, \eta \in \mathbb{R}^{d}$.

Proof. By hypothesis $C$ takes values in $\Sigma_{\theta}$. This implies $((\operatorname{Re} C) \xi, \xi) \geq 0$ for all $\xi \in \mathbb{C}^{d}$. We deduce that $\left(R_{s} \xi, \xi\right) \geq 0$ for all $\xi \in \mathbb{R}^{d}$. Finally we use polarisation to obtain the lemma.

Lemma 2.2. We have

$$
\left|\left(B_{s} \xi, \eta\right)\right| \leq \frac{1}{2}(\tan \theta)\left(\left(R_{s} \xi, \xi\right)+\left(R_{s} \eta, \eta\right)\right)
$$

for all $\xi, \eta \in \mathbb{R}^{d}$. 
Proof. Since $C$ takes values in $\Sigma_{\theta}$, we have

$$
|((\operatorname{Im} C) \xi, \xi)| \leq(\tan \theta)((\operatorname{Re} C) \xi, \xi)
$$

for all $\xi \in \mathbb{C}^{d}$. It follows that

$$
\left|\left(B_{s} \xi, \xi\right)\right| \leq(\tan \theta)\left(R_{s} \xi, \xi\right)
$$

for all $\xi \in \mathbb{R}^{d}$. Finally we use polarisation to obtain

$$
\left|\left(B_{s} \xi, \eta\right)\right| \leq(\tan \theta)\left(R_{s} \xi, \xi\right)^{1 / 2}\left(R_{s} \eta, \eta\right)^{1 / 2} \leq \frac{1}{2}(\tan \theta)\left(\left(R_{s} \xi, \xi\right)+\left(R_{s} \eta, \eta\right)\right)
$$

for all $\xi, \eta \in \mathbb{R}^{d}$ as required.

Lemma 2.3. We have

$$
\left|\left(B_{s} \xi, \xi\right)+\left(B_{s} \eta, \eta\right)-2\left(R_{a} \xi, \eta\right)\right| \leq(\tan \theta)\left(\left(R_{s} \xi, \xi\right)+\left(R_{s} \eta, \eta\right)+2\left(B_{a} \xi, \eta\right)\right)
$$

for all $\xi, \eta \in \mathbb{R}^{d}$.

Proof. Let $\xi, \eta \in \mathbb{R}^{d}$. Then

$$
((\operatorname{Im} C)(\xi+i \eta), \xi+i \eta)=\left(B_{s} \xi, \xi\right)+\left(B_{s} \eta, \eta\right)-2\left(R_{a} \xi, \eta\right)
$$

and

$$
((\operatorname{Re} C)(\xi+i \eta), \xi+i \eta)=\left(R_{s} \xi, \xi\right)+\left(R_{s} \eta, \eta\right)+2\left(B_{a} \xi, \eta\right) .
$$

The claim is now immediate from (11).

Lemma 2.4. Suppose $B_{a}=0$. Then

$$
\left|\left(R_{a} \xi, \eta\right)\right| \leq(\tan \theta)\left(\left(R_{s} \xi, \xi\right)+\left(R_{s} \eta, \eta\right)\right)
$$

for all $\xi, \eta \in \mathbb{R}^{d}$.

Proof. Since $B_{a}=0$, Lemma 2.3 gives

$$
\left|\left(B_{s} \xi, \xi\right)+\left(B_{s} \eta, \eta\right)-2\left(R_{a} \xi, \eta\right)\right| \leq(\tan \theta)\left(\left(R_{s} \xi, \xi\right)+\left(R_{s} \eta, \eta\right)\right) .
$$

The result now follows from the triangle inequality and Lemma 2.2.

Lemma 2.5. Let $Q$ be a positive matrix and $U$ a complex $d \times d$ matrix. Then

$$
(Q U \xi, U \xi) \leq \operatorname{tr}\left(U^{*} Q U\right)\|\xi\|^{2}
$$

for all $\xi \in \mathbb{C}^{d}$.

Proof. Since $Q$ is a positive matrix, we have $(Q U \xi, U \xi) \geq 0$ for all $\xi \in \mathbb{C}^{d}$. It follows that $U^{*} Q U \geq 0$. Hence $U^{*} Q U \leq \operatorname{tr}\left(U^{*} Q U\right) I$, where $I$ denotes the identity matrix. This justifies the claim.

Lemma 2.6. We have the following.

(a) $\left(R_{s} \xi, \xi\right) \geq 0$ for all $\xi \in \mathbb{C}^{d}$. 
(b) $\quad\left(\left((\tan \theta) R_{s} \pm B_{s}\right) \xi, \xi\right) \geq 0$ for all $\xi \in \mathbb{C}^{d}$.

(c) Suppose $B_{a}=0$. Then $\left(\left(2(\tan \theta) R_{s} \pm i R_{a}\right) \xi, \xi\right) \geq 0$ for all $\xi \in \mathbb{C}^{d}$.

Proof. Let $\xi \in \mathbb{C}^{d}$. Write $\xi=\xi_{1}+i \xi_{2}$, where $\xi_{1}, \xi_{2} \in \mathbb{R}^{d}$. We note that

$$
\left(R_{s} \xi, \xi\right)=\left(R_{s} \xi_{1}, \xi_{1}\right)+\left(R_{s} \xi_{2}, \xi_{2}\right)
$$

and

$$
\left(B_{s} \xi, \xi\right)=\left(B_{s} \xi_{1}, \xi_{1}\right)+\left(B_{s} \xi_{2}, \xi_{2}\right)
$$

Also

$$
\left(R_{a} \xi, \xi\right)=-2 i\left(R_{a} \xi_{1}, \xi_{2}\right) .
$$

The claim now follows from Lemmas 2.1, 2.2 and 2.4.

Next let $\alpha \in\left(-\frac{\pi}{2}+\theta, \frac{\pi}{2}-\theta\right)$ and write $C_{\alpha}=e^{i \alpha} C$. In a similar manner as above, we define $\operatorname{Re}\left(C_{\alpha}\right), \operatorname{Im}\left(C_{\alpha}\right), R_{\alpha}, B_{\alpha}, R_{s, \alpha}, R_{a, \alpha}, B_{s, \alpha}$ and $B_{a, \alpha}$. Note that we also have

$$
\operatorname{Re}\left(C_{\alpha}\right)=R_{s, \alpha}+i B_{a, \alpha} \quad \text { and } \quad \operatorname{Im}\left(C_{\alpha}\right)=B_{s, \alpha}-i R_{a, \alpha} .
$$

Lemma 2.7. Let $j \in\{1, \ldots, d\}$. Suppose $U$ is a complex $d \times d$ matrix with $U^{T}=U$. Then

$$
\left|\operatorname{tr}\left(\left(\partial_{j} C_{\alpha}\right) U\right)\right|^{2} \leq M \operatorname{tr}\left(U R_{s, \alpha} \bar{U}\right),
$$

where

$$
M=32 d(1+\tan (\theta+\alpha))^{2}\left\|\partial_{l}^{2} C\right\|_{\infty} .
$$

Proof. It follows from [Do16a, Corollary 2.6] that

$$
\begin{aligned}
\left|\operatorname{tr}\left(\left(\partial_{j} C_{\alpha}\right) U\right)\right|^{2} & \leq 32 d(1+\tan (\theta+\alpha))^{2}\left\|\partial_{l}^{2}\left(e^{i \alpha} C\right)\right\|_{\infty} \operatorname{tr}\left(U R_{s, \alpha} \bar{U}\right) \\
& \leq 32 d(1+\tan (\theta+\alpha))^{2}\left\|\partial_{l}^{2} C\right\|_{\infty} \operatorname{tr}\left(U R_{s, \alpha} \bar{U}\right)
\end{aligned}
$$

as required.

Lemma 2.8. Suppose $B_{a}=0$. Then the following hold.

(i) $\operatorname{Re}\left(C_{\alpha}\right)=R_{s} \cos \alpha-B_{s} \sin \alpha+i R_{a} \sin \alpha$.

(ii) $\operatorname{Im}\left(C_{\alpha}\right)=R_{s} \sin \alpha+B_{s} \cos \alpha-i R_{a} \cos \alpha$.

(iii) $R_{\alpha}=R_{s} \cos \alpha+R_{a} \cos \alpha-B_{s} \sin \alpha$,

$R_{s, \alpha}=R_{s} \cos \alpha-B_{s} \sin \alpha$,

$R_{a, \alpha}=R_{a} \cos \alpha$.

(iv) $B_{\alpha}=R_{s} \sin \alpha+R_{a} \sin \alpha+B_{s} \cos \alpha$,

$B_{s, \alpha}=R_{s} \sin \alpha+B_{s} \cos \alpha$,

$B_{a, \alpha}=R_{a} \sin \alpha$.

Proof. These identities follow directly from the definition of $C$ and $C_{\alpha}$. 


\section{Sectorial property}

Let $p \in(1, \infty)$. Let $\Omega, \theta, C$ and $A_{p}$ be as in Section 1, In this section we prove Theorem 1.2. A convenient tool that we will use repeatedly is the formula of integration by parts in Sobolev spaces given in the next theorem. The theorem is immediate from the proof of [MS08, Proposition 3.5]. We emphasise that we do not require $C=C^{T}$ in this theorem (cf. [MS08, Theorem 3.1] for the same result but with extra assumption that $C=C^{T}$ ).

Theorem 3.1. Let $u \in D\left(A_{p}\right)$. Then

$$
\begin{aligned}
\int_{[u \neq 0]}\left(A_{p} u\right)|u|^{p-2} \bar{u}= & \int_{[u \neq 0]}|u|^{p-2}(C \nabla \bar{u}, \nabla \bar{u}) \\
& +(p-2) \int_{[u \neq 0]}|u|^{p-4}(C \operatorname{Re}(u \nabla \bar{u}), \operatorname{Re}(u \nabla \bar{u})) \\
& -i(p-2) \int_{[u \neq 0]}|u|^{p-4}(C \operatorname{Re}(u \nabla \bar{u}), \operatorname{Im}(u \nabla \bar{u})) .
\end{aligned}
$$

Using Theorem 3.1 we obtain the following.

Proposition 3.2. Let $u \in D\left(A_{p}\right)$. Write $u \nabla \bar{u}=\xi+i \eta$, where $\xi, \eta \in \mathbb{R}^{d}$. Then

$$
\begin{aligned}
\operatorname{Re}\left(A_{p} u,|u|^{p-2} u \mathbb{1}_{[u \neq 0]}\right)=\int_{[u \neq 0]}|u|^{p-4} & \left((p-1)\left(R_{s} \xi, \xi\right)+\left(R_{s} \eta, \eta\right)\right. \\
& \left.+(p-2)\left(B_{s} \xi, \eta\right)+p\left(B_{a} \xi, \eta\right)\right)
\end{aligned}
$$

and

$$
\begin{aligned}
\operatorname{Im}\left(A_{p} u,|u|^{p-2} u \mathbb{1}_{[u \neq 0]}\right)=\int_{[u \neq 0]}|u|^{p-4} & ( \\
& (p-1)\left(B_{s} \xi, \xi\right)+\left(B_{s} \eta, \eta\right) \\
& \left.-(p-2)\left(R_{s} \xi, \eta\right)-p\left(R_{a} \xi, \eta\right)\right) .
\end{aligned}
$$

Proof. We will prove the first inequality only. The second is similar.

Consider (12). We have

$$
\begin{aligned}
|u|^{2}(C \nabla \bar{u}, \nabla \bar{u})= & (C u \nabla \bar{u}, u \nabla \bar{u})=(C(\xi+i \eta), \xi+i \eta) \\
= & (R \xi, \xi)+(R \eta, \eta)+(B \xi, \eta)-(B \eta, \xi) \\
& -i((R \eta, \xi)-(R \xi, \eta)+(B \xi, \xi)+(B \eta, \eta)) .
\end{aligned}
$$

Therefore

$$
\begin{aligned}
\operatorname{Re}\left(|u|^{2}(C \nabla \bar{u}, \nabla \bar{u})\right) & =(R \xi, \xi)+(R \eta, \eta)+(B \xi, \eta)-(B \eta, \xi) \\
& =\left(R_{s} \xi, \xi\right)+\left(R_{s} \eta, \eta\right)+2\left(B_{a} \xi, \eta\right) .
\end{aligned}
$$

Also

$$
\operatorname{Re}(C \operatorname{Re}(u \nabla \bar{u}), \operatorname{Re}(u \nabla \bar{u}))=\operatorname{Re}(C \xi, \xi)=(R \xi, \xi)=\left(R_{s} \xi, \xi\right) .
$$

Similarly

$$
\operatorname{Re}(i(C \operatorname{Re}(u \nabla \bar{u}), \operatorname{Im}(u \nabla \bar{u})))=\operatorname{Re}(i(C \xi, \eta))=-(B \xi, \eta)=-\left(B_{s} \xi, \eta\right)-\left(B_{a} \xi, \eta\right) .
$$

Hence taking the real parts on both sides of (12) yields the result. 
The following lemma is essential in the proof of Theorem 1.2.

Lemma 3.3. Suppose $\left|1-\frac{2}{p}\right|<\cos \theta$. Let $\phi=\arccos \left|1-\frac{2}{p}\right|$. Then

$$
\begin{aligned}
& \left(\tan \left(\frac{\pi}{2}-\phi\right)+\tan \theta\right)\left(\left(R_{s} \xi, \xi\right)+\left(R_{s} \eta, \eta\right)\right) \\
\leq & \tan \left(\frac{\pi}{2}-\phi+\theta\right)\left(\left(R_{s} \xi, \xi\right)+\left(R_{s} \eta, \eta\right)+\frac{p-2}{\sqrt{p-1}}\left(B_{s} \xi, \eta\right)\right)
\end{aligned}
$$

for all $\xi, \eta \in \mathbb{R}^{d}$.

Proof. First note that

$$
\begin{aligned}
& \tan \left(\frac{\pi}{2}-\phi\right)(\tan \theta)\left(\left(R_{s} \xi, \xi\right)+\left(R_{s} \eta, \eta\right)\right)+\frac{p-2}{\sqrt{p-1}}\left(B_{s} \xi^{\prime}, \eta\right) \\
\geq & \tan \left(\frac{\pi}{2}-\phi\right)(\tan \theta)\left(\left(R_{s} \xi, \xi\right)+\left(R_{s} \eta, \eta\right)\right)-\frac{|p-2|}{\sqrt{p-1}}\left|\left(B_{s} \xi, \eta\right)\right| \\
= & \tan \left(\frac{\pi}{2}-\phi\right)\left((\tan \theta)\left(\left(R_{s} \xi, \xi\right)+\left(R_{s} \eta, \eta\right)\right)-2\left|\left(B_{s} \xi, \eta\right)\right|\right) \geq 0
\end{aligned}
$$

as $\tan \left(\frac{\pi}{2}-\phi\right)=\cot (\phi)=\frac{|p-2|}{2 \sqrt{p-1}}$ and we used Lemma 2.2 in the last step. We also deduce from the hypotheses that $\tan \left(\frac{\pi}{2}-\phi+\theta\right) \geq 0$. Therefore

$$
\begin{aligned}
& \left(\tan \left(\frac{\pi}{2}-\phi\right)+\tan \theta\right)\left(\left(R_{s} \xi, \xi\right)+\left(R_{s} \eta, \eta\right)\right) \\
\leq & \left(\tan \left(\frac{\pi}{2}-\phi\right)+\tan \theta\right)\left(\left(R_{s} \xi, \xi\right)+\left(R_{s} \eta, \eta\right)\right) \\
& \quad+\tan \left(\frac{\pi}{2}-\phi+\theta\right)\left(\tan \left(\frac{\pi}{2}-\phi\right)(\tan \theta)\left(\left(R_{s} \xi, \xi\right)+\left(R_{s} \eta, \eta\right)\right)+\frac{p-2}{\sqrt{p-1}}\left(B_{s} \xi, \eta\right)\right) \\
= & \tan \left(\frac{\pi}{2}-\phi+\theta\right)\left(\left(R_{s} \xi, \xi\right)+\left(R_{s} \eta, \eta\right)+\frac{p-2}{\sqrt{p-1}}\left(B_{s} \xi, \eta\right)\right),
\end{aligned}
$$

where we used (13) in the first step.

Next we prove Theorem 1.2.

Proof of Theorem 1.2. Let $u \in D\left(A_{p}\right)$. Write $u \nabla \bar{u}=\xi+i \eta$, where $\xi, \eta \in \mathbb{R}^{d}$. By Proposition 3.2, it suffices to show that

$$
\begin{aligned}
& \left|(p-1)\left(B_{s} \xi, \xi\right)+\left(B_{s} \eta, \eta\right)-(p-2)\left(R_{s} \xi, \eta\right)-p\left(R_{a} \xi, \eta\right)\right| \\
\leq & K\left((p-1)\left(R_{s} \xi, \xi\right)+\left(R_{s} \eta, \eta\right)+(p-2)\left(B_{s} \xi, \eta\right)\right),
\end{aligned}
$$

where $K$ is defined by (5). Set $\xi^{\prime}=\sqrt{p-1} \xi$. Then (14) is equivalent to

$$
\begin{aligned}
& \left|\left(B_{s} \xi^{\prime}, \xi^{\prime}\right)+\left(B_{s} \eta, \eta\right)-\frac{p-2}{\sqrt{p-1}}\left(R_{s} \xi^{\prime}, \eta\right)-\frac{p}{\sqrt{p-1}}\left(R_{a} \xi^{\prime}, \eta\right)\right| \\
\leq & K\left(\left(R_{s} \xi^{\prime}, \xi^{\prime}\right)+\left(R_{s} \eta, \eta\right)+\frac{p-2}{\sqrt{p-1}}\left(B_{s} \xi^{\prime}, \eta\right)\right) .
\end{aligned}
$$

Note that by Lemma 2.1 we have

$$
\frac{|p-2|}{\sqrt{p-1}}\left|\left(R_{s} \xi^{\prime}, \eta\right)\right| \leq \tan \left(\frac{\pi}{2}-\phi\right)\left(\left(R_{s} \xi^{\prime}, \xi^{\prime}\right)+\left(R_{s} \eta, \eta\right)\right)
$$


as $\tan \left(\frac{\pi}{2}-\phi\right)=\cot (\phi)=\frac{|p-2|}{2 \sqrt{p-1}}$.

Now we consider two cases.

Case 1: Suppose $R_{a}=0$. Using Lemma 2.2 again we obtain

$$
\left|\left(B_{s} \xi^{\prime}, \xi^{\prime}\right)+\left(B_{s} \eta, \eta\right)\right| \leq(\tan \theta)\left(\left(R_{s} \xi^{\prime}, \xi^{\prime}\right)+\left(R_{s} \eta, \eta\right)\right)
$$

It follows that

$$
\begin{aligned}
& \left|\left(B_{s} \xi^{\prime}, \xi^{\prime}\right)+\left(B_{s} \eta, \eta\right)-\frac{p-2}{\sqrt{p-1}}\left(R_{s} \xi^{\prime}, \eta\right)-\frac{p}{\sqrt{p-1}}\left(R_{a} \xi^{\prime}, \eta\right)\right| \\
= & \left|\left(B_{s} \xi^{\prime}, \xi^{\prime}\right)+\left(B_{s} \eta, \eta\right)-\frac{p-2}{\sqrt{p-1}}\left(R_{s} \xi^{\prime}, \eta\right)\right| \\
\leq & \left(\tan \left(\frac{\pi}{2}-\phi\right)+\tan \theta\right)\left(\left(R_{s} \xi^{\prime}, \xi^{\prime}\right)+\left(R_{s} \eta, \eta\right)\right) \\
\leq & \tan \left(\frac{\pi}{2}-\phi+\theta\right)\left(\left(R_{s} \xi^{\prime}, \xi^{\prime}\right)+\left(R_{s} \eta, \eta\right)+\frac{p-2}{\sqrt{p-1}}\left(B_{s} \xi^{\prime}, \eta\right)\right),
\end{aligned}
$$

where we used $R_{a}=0$ in the first step, (17) and (16) in the second step and Lemma 3.3 in the last step.

Hence (15) is valid and the result follows in this case.

Case 2: Suppose $R_{a} \neq 0$. We rewrite the left hand side of (15) as

$$
\begin{aligned}
L:= & \mid\left(\left(B_{s} \xi^{\prime}, \xi^{\prime}\right)+\left(B_{s} \eta, \eta\right)-2\left(R_{a} \xi^{\prime}, \eta\right)\right)-\frac{p-2}{\sqrt{p-1}}\left(R_{s} \xi^{\prime}, \eta\right) \\
& -\left(\frac{p}{\sqrt{p-1}}-2\right)\left(R_{a} \xi^{\prime}, \eta\right) \mid .
\end{aligned}
$$

(Note that $\frac{p}{\sqrt{p-1}} \geq 2$ for all $p \in(1, \infty)$.) Since $B_{a}=0$, it follows from Lemma 2.3 that

$$
\left|\left(B_{s} \xi^{\prime}, \xi^{\prime}\right)+\left(B_{s} \eta, \eta\right)-2\left(R_{a} \xi^{\prime}, \eta\right)\right| \leq(\tan \theta)\left(\left(R_{s} \xi^{\prime}, \xi^{\prime}\right)+\left(R_{s} \eta, \eta\right)\right) .
$$

Next we deduce from Lemma 2.4 that

$$
\left(\frac{p}{\sqrt{p-1}}-2\right)\left|\left(R_{a} \xi^{\prime}, \eta\right)\right| \leq\left(\frac{2}{\sin \phi}-2\right)(\tan \theta)\left(\left(R_{s} \xi^{\prime}, \xi^{\prime}\right)+\left(R_{s} \eta, \eta\right)\right)
$$

as $\sin \phi=\frac{2 \sqrt{p-1}}{p}$. Now it follows from (16), (18) and (19) that

$$
\begin{aligned}
L \leq & \left(\left(\frac{2}{\sin \phi}-1\right) \tan \theta+\tan \left(\frac{\pi}{2}-\phi\right)\right)\left(\left(R_{s} \xi^{\prime}, \xi^{\prime}\right)+\left(R_{s} \eta, \eta\right)\right) \\
= & \frac{\left(\frac{2}{\sin \phi}-1\right) \tan \theta+\tan \left(\frac{\pi}{2}-\phi\right)}{\tan \theta+\tan \left(\frac{\pi}{2}-\phi\right)}\left(\tan \theta+\tan \left(\frac{\pi}{2}-\phi\right)\right)\left(\left(R_{s} \xi^{\prime}, \xi^{\prime}\right)+\left(R_{s} \eta, \eta\right)\right) \\
\leq & \frac{\left(\frac{2}{\sin \phi}-1\right) \tan \theta+\tan \left(\frac{\pi}{2}-\phi\right)}{\tan \theta+\tan \left(\frac{\pi}{2}-\phi\right)} \tan \left(\frac{\pi}{2}-\phi+\theta\right)\left(\left(R_{s} \xi^{\prime}, \xi^{\prime}\right)+\left(R_{s} \eta, \eta\right)\right. \\
& \left.+\frac{p-2}{\sqrt{p-1}}\left(B_{s} \xi^{\prime}, \eta\right)\right) \\
= & \frac{\left(\frac{2}{\sin \phi}-1\right) \tan \theta+\tan \left(\frac{\pi}{2}-\phi\right)}{1-(\tan \theta) \tan \left(\frac{\pi}{2}-\phi\right)}\left(\left(R_{s} \xi^{\prime}, \xi^{\prime}\right)+\left(R_{s} \eta, \eta\right)+\frac{p-2}{\sqrt{p-1}}\left(B_{s} \xi^{\prime}, \eta\right)\right),
\end{aligned}
$$

where we used Lemma 3.3 in the second step.

Hence (15) is also valid in this case. 


\section{Generation of contraction holomorphic semigroup}

Let $\Omega=\mathbb{R}^{d}$ and $\theta \in\left[0, \frac{\pi}{2}\right)$. We assume $c_{k l} \in W^{2, \infty}\left(\mathbb{R}^{d}, \mathbb{C}\right)$ for all $k, l \in\{1, \ldots, d\}$. Assume further that $(C(x) \xi, \xi) \in \Sigma_{\theta}$ for all $x \in \mathbb{R}^{d}$ and $\xi \in \mathbb{C}^{d}$, where $C=\left(c_{k l}\right)_{1 \leq k, l \leq d}$ and $\Sigma_{\theta}$ is defined by (2) .

Let $p \in(1, \infty)$. We will prove in Proposition 4.1 that $A_{p}$ is closable. Let $\overline{A_{p}}$ be the closure of $A_{p}$. We will show in this section that $-\overline{A_{p}}$ generates a holomorphic semigroup on $L_{p}\left(\mathbb{R}^{d}\right)$ which is contractive on a sector. This is the content of Theorems 1.3 and 1.4.

First we introduce some more definitions. Let $q$ be such that $\frac{1}{p}+\frac{1}{q}=1$. Define

$$
H_{q} u=-\sum_{k, l=1}^{d} \partial_{k}\left(\overline{c_{k l}} \partial_{l} u\right)
$$

on the domain

$$
D\left(H_{q}\right)=C_{c}^{\infty}\left(\mathbb{R}^{d}\right)
$$

Define

$$
B_{p}=\left(H_{q}\right)^{*},
$$

which is the dual of $H_{q}$. Then $B_{p}$ is closed by [Kat80, Theorem III.5.29]. Also note that $W^{2, p}\left(\mathbb{R}^{d}\right) \subset D\left(B_{p}\right)$ and

$$
B_{p} u=-\sum_{k, l=1}^{d} \partial_{l}\left(c_{k l} \partial_{k} u\right)
$$

for all $u \in W^{2, p}\left(\mathbb{R}^{d}\right)$.

Proposition 4.1. The operator $A_{p}$ is closable.

Proof. Since $A_{p} \subset B_{p}$ and $B_{p}$ is closed, the operator $A_{p}$ is closable. tion.

It turns out that $B_{p}=\overline{A_{p}}$ under certain conditions, as shown in the following proposi-

Proposition 4.2. Suppose $\left|1-\frac{2}{p}\right| \leq \cos \theta$ and $B_{a}=0$. Then $\overline{A_{p}}=B_{p}$. Moreover, $\overline{A_{p}}$ is $m$-accretive.

Proof. By [Do16a, Proposition 4.9] the operator $B_{p}$ is $m$-accretive and the space $C_{c}^{\infty}\left(\mathbb{R}^{d}\right)$ of test functions is a core for $B_{p}$. It follows that $\overline{A_{p}}=B_{p}$ and $A_{p}$ is $m$-accretive as claimed.

Using Theorem 1.2 we are now able to prove the generation result in Theorem 1.3,

Proof of Theorem 1.3. It follows from Theorem 1.2 that

$$
\left|\operatorname{Im}\left(\overline{A_{p}} u,|u|^{p-2} u \mathbb{1}_{[u \neq 0]}\right)\right| \leq K \operatorname{Re}\left(\overline{A_{p}} u,|u|^{p-2} u \mathbb{1}_{[u \neq 0]}\right)
$$

for all $u \in D\left(\overline{A_{p}}\right)$, where $K$ is defined by (5). Therefore the interior $\Sigma_{\pi-\arctan (K)}^{\circ} \subset \rho\left(-\overline{A_{p}}\right)$ by [Paz83, Theorem 1.3.9] and Proposition 4.2, where $\rho\left(-\overline{A_{p}}\right)$ denotes the resolvent set of $-\overline{A_{p}}$. Moreover,

$$
\left\|\left(\lambda+\overline{A_{p}}\right)^{-1}\right\|_{p \rightarrow p} \leq \frac{1}{\operatorname{dist}\left(\lambda, S\left(-\overline{A_{p}}\right)\right)}
$$


for all $\lambda \in \Sigma_{\pi-\arctan (K)}^{\circ}$, where $S\left(-\overline{A_{p}}\right)$ is the numerical range of $-\overline{A_{p}}$ defined by

$$
S\left(-\overline{A_{p}}\right)=\left\{-\left(\overline{A_{p}} u,|u|^{p-2} u \mathbb{1}_{[u \neq 0]}\right): u \in D\left(\overline{A_{p}}\right) \text { and }\|u\|_{p}=1\right\} .
$$

Let $\varepsilon \in(0, \pi-\arctan (K))$. Then dist $\left(\lambda, S\left(-\overline{A_{p}}\right)\right) \geq(\sin \varepsilon)|\lambda|$ for all $\lambda \in \Sigma_{\pi-\arctan (K)-\varepsilon}$. Therefore (21) implies

$$
\left\|\left(\lambda+\overline{A_{p}}\right)^{-1}\right\|_{p \rightarrow p} \leq \frac{1}{(\sin \varepsilon)|\lambda|}
$$

for all $\lambda \in \Sigma_{\pi-\arctan (K)-\varepsilon}$. Hence we deduce from [Paz83, Theorem 2.5.2(c)] that $-\overline{A_{p}}$ generates a holomorphic semigroup on $L_{p}\left(\mathbb{R}^{d}\right)$ with $\operatorname{angle} \psi=\frac{\pi}{2}-\arctan (K)$.

Our next aim is to show Theorem 1.4. We will do this by first showing that $-B_{p}$ generates a holomorphic semigroup which is contractive on a sector. This together with Proposition 4.2 imply the theorem. We first obtain some preliminary results.

In what follows we let $B_{p, \alpha}=e^{i \alpha} B_{p}$ for all $\alpha \in\left(-\frac{\pi}{2}+\theta, \frac{\pi}{2}-\theta\right)$ and adopt the notation used in Lemmas 2.7 and 2.8. We aim to show that $B_{p, \alpha}$ is an $m$-accretive operator for all $\alpha$ in a suitable range. Following [WD83] and [Do16a] we need two crucial inequalities for $B_{p, \alpha}$ in order to do this. The first inequality is given by the next proposition. The second inequality is derived in Proposition 4.5.

Proposition 4.3. Suppose $B_{a}=0$. Let $p \in(1, \infty)$ be such that $\left|1-\frac{2}{p}\right|<\cos \theta$. Let $\alpha \in(-\psi, \psi)$, where $\psi$ is given by (6). Then

$$
\operatorname{Re}\left(B_{p, \alpha} u,|u|^{p-2} u \mathbb{1}_{[u \neq 0]}\right) \geq 0
$$

for all $u \in W^{2, p}\left(\mathbb{R}^{d}\right)$.

Proof. Let $u \in W^{2, p}\left(\mathbb{R}^{d}\right)$. It follows from Theorem 3.1 that

$$
\begin{aligned}
\left(B_{p, \alpha} u,|u|^{p-2} u \mathbb{1}_{[u \neq 0]}\right)= & \int_{[u \neq 0]}|u|^{p-2}\left(C_{\alpha} \nabla \bar{u}, \nabla \bar{u}\right) \\
& +(p-2) \int_{[u \neq 0]}|u|^{p-4}\left(C_{\alpha} \operatorname{Re}(u \nabla \bar{u}), \operatorname{Re}(u \nabla \bar{u})\right) \\
& -i(p-2) \int_{[u \neq 0]}|u|^{p-4}\left(C_{\alpha} \operatorname{Re}(u \nabla \bar{u}), \operatorname{Im}(u \nabla \bar{u})\right) .
\end{aligned}
$$

Write $u \nabla \bar{u}=\xi+i \eta$, where $\xi, \eta \in \mathbb{R}^{d}$. Then

$$
\begin{aligned}
|u|^{2}\left(C_{\alpha} \nabla \bar{u}, \nabla \bar{u}\right)= & \left(C_{\alpha} u \nabla \bar{u}, u \nabla \bar{u}\right)=\left(C_{\alpha}(\xi+i \eta), \xi+i \eta\right) \\
= & \left(R_{\alpha} \xi, \xi\right)+\left(R_{\alpha} \eta, \eta\right)+\left(B_{\alpha} \xi, \eta\right)-\left(B_{\alpha} \eta, \xi\right) \\
& +i\left(\left(R_{\alpha} \eta, \xi\right)-\left(R_{\alpha} \xi, \eta\right)+\left(B_{\alpha} \xi, \xi\right)+\left(B_{\alpha} \eta, \eta\right)\right) .
\end{aligned}
$$

Therefore

$$
\begin{aligned}
\operatorname{Re}\left(|u|^{2}\left(C_{\alpha} \nabla \bar{u}, \nabla \bar{u}\right)\right) & =\left(R_{\alpha} \xi, \xi\right)+\left(R_{\alpha} \eta, \eta\right)+\left(B_{\alpha} \xi, \eta\right)-\left(B_{\alpha} \eta, \xi\right) \\
& =\left(R_{s, \alpha} \xi, \xi\right)+\left(R_{s, \alpha} \eta, \eta\right)+2\left(B_{a, \alpha} \xi, \eta\right)
\end{aligned}
$$

We also have

$$
\operatorname{Re}\left(C_{\alpha} \operatorname{Re}(u \nabla \bar{u}), \operatorname{Re}(u \nabla \bar{u})\right)=\operatorname{Re}\left(C_{\alpha} \xi, \xi\right)=\left(R_{\alpha} \xi, \xi\right)=\left(R_{s, \alpha} \xi, \xi\right) .
$$


Similarly

$\operatorname{Re}\left(i\left(C_{\alpha} \operatorname{Re}(u \nabla \bar{u}), \operatorname{Im}(u \nabla \bar{u})\right)\right)=\operatorname{Re}\left(i\left(C_{\alpha} \xi, \eta\right)\right)=-\left(B_{\alpha} \xi, \eta\right)=-\left(B_{s, \alpha} \xi, \eta\right)-\left(B_{a, \alpha} \xi, \eta\right)$.

Hence taking the real parts on both sides of (22) yields

$$
\begin{aligned}
& \operatorname{Re}\left(B_{p, \alpha} u,|u|^{p-2} u \mathbb{1}_{[u \neq 0]}\right) \\
= & \int_{[u \neq 0]}|u|^{p-4}\left((p-1)\left(R_{s, \alpha} \xi, \xi\right)+\left(R_{s, \alpha} \eta, \eta\right)+p\left(B_{a, \alpha} \xi, \eta\right)+(p-2)\left(B_{s, \alpha} \xi, \eta\right)\right) \\
= & \int_{[u \neq 0]}|u|^{p-4}\left(\left(R_{s, \alpha} \xi^{\prime}, \xi^{\prime}\right)+\left(R_{s, \alpha} \eta, \eta\right)+\frac{p}{\sqrt{p-1}}\left(B_{a, \alpha} \xi^{\prime}, \eta\right)+\frac{p-2}{\sqrt{p-1}}\left(B_{s, \alpha} \xi^{\prime}, \eta\right)\right),
\end{aligned}
$$

where $\xi^{\prime}=\sqrt{p-1} \xi$. Set

$$
P=\left(R_{s, \alpha} \xi^{\prime}, \xi^{\prime}\right)+\left(R_{s, \alpha} \eta, \eta\right)+\frac{p}{\sqrt{p-1}}\left(B_{a, \alpha} \xi^{\prime}, \eta\right)+\frac{p-2}{\sqrt{p-1}}\left(B_{s, \alpha} \xi^{\prime}, \eta\right) .
$$

We will show that $P \geq 0$. We consider 2 cases.

Case 1: Suppose $R_{a}=0$. Note that $\cot \phi=\frac{|p-2|}{2 \sqrt{p-1}}$. We have

$$
\left|(\sin \alpha)\left(\left(B_{s} \xi^{\prime}, \xi^{\prime}\right)+\left(B_{s} \eta, \eta\right)\right)\right| \leq \sin (|\alpha|)(\tan \theta)\left(\left(R_{s} \xi^{\prime}, \xi^{\prime}\right)+\left(R_{s} \eta, \eta\right)\right)
$$

and

$$
\left|\frac{p-2}{\sqrt{p-1}}(\cos \alpha)\left(B_{s} \xi^{\prime}, \eta\right)\right| \leq(\cot \phi)(\cos \alpha)(\tan \theta)\left(\left(R_{s} \xi^{\prime}, \xi^{\prime}\right)+\left(R_{s} \eta, \eta\right)\right) .
$$

by Lemma 2.2. Also

$$
\left|\frac{p-2}{\sqrt{p-1}}(\sin \alpha)\left(R_{s} \xi^{\prime}, \eta\right)\right| \leq(\cot \phi) \sin (|\alpha|)\left(\left(R_{s} \xi^{\prime}, \xi^{\prime}\right)+\left(R_{s} \eta, \eta\right)\right)
$$

by Lemma 2.1. Since $R_{a}=0$, Lemma 2.8) gives $B_{a, \alpha}=(\sin \alpha) R_{a}=0$. It follows from Lemma 2.8, (24), (25), (26) and (27) that

$$
\begin{aligned}
P= & \left(R_{s, \alpha} \xi^{\prime}, \xi^{\prime}\right)+\left(R_{s, \alpha} \eta, \eta\right)+\frac{p-2}{\sqrt{p-1}}\left(B_{s, \alpha} \xi^{\prime}, \eta\right) \\
= & (\cos \alpha)\left(\left(R_{s} \xi^{\prime}, \xi^{\prime}\right)+\left(R_{s} \eta, \eta\right)\right)-(\sin \alpha)\left(\left(B_{s} \xi^{\prime}, \xi^{\prime}\right)+\left(B_{s} \eta, \eta\right)\right) \\
& \quad+\frac{p-2}{\sqrt{p-1}}(\sin \alpha)\left(R_{s} \xi^{\prime}, \eta\right)+\frac{p-2}{\sqrt{p-1}}(\cos \alpha)\left(B_{s} \xi^{\prime}, \eta\right) \\
\geq & (\cos \alpha-\sin (|\alpha|) \tan \theta-(\cot \phi) \sin (|\alpha|)-(\cot \phi)(\cos \alpha) \tan \theta)\left(\left(R_{s} \xi^{\prime}, \xi^{\prime}\right)+\left(R_{s} \eta, \eta\right)\right) \\
\geq & 0,
\end{aligned}
$$

where we used the fact that $\alpha \in(-\psi, \psi)$ in the last step. Hence we deduce from (23) that $\operatorname{Re}\left(B_{p, \alpha} u,|u|^{p-2} u \mathbb{1}_{[u \neq 0]}\right) \geq 0$ in this case. 
Case 2: Suppose $R_{a} \neq 0$. Expanding (24) using Lemma 2.8 gives

$$
\begin{aligned}
P= & \left(R_{s, \alpha} \xi^{\prime}, \xi^{\prime}\right)+\left(R_{s, \alpha} \eta, \eta\right)+\frac{p}{\sqrt{p-1}}\left(B_{a, \alpha} \xi^{\prime}, \eta\right)+\frac{p-2}{\sqrt{p-1}}\left(B_{s, \alpha} \xi^{\prime}, \eta\right) \\
= & (\cos \alpha)\left(\left(R_{s} \xi^{\prime}, \xi^{\prime}\right)+\left(R_{s} \eta, \eta\right)\right)-(\sin \alpha)\left(\left(B_{s} \xi^{\prime}, \xi^{\prime}\right)+\left(B_{s} \eta, \eta\right)\right) \\
& +\frac{p}{\sqrt{p-1}}(\sin \alpha)\left(R_{a} \xi^{\prime}, \eta\right)+\frac{p-2}{\sqrt{p-1}}(\sin \alpha)\left(R_{s} \xi^{\prime}, \eta\right)+\frac{p-2}{\sqrt{p-1}}(\cos \alpha)\left(B_{s} \xi^{\prime}, \eta\right) \\
= & (\cos \alpha)\left(\left(R_{s} \xi^{\prime}, \xi^{\prime}\right)+\left(R_{s} \eta, \eta\right)\right)-(\sin \alpha)\left(\left(B_{s} \xi^{\prime}, \xi^{\prime}\right)+\left(B_{s} \eta, \eta\right)-2\left(R_{a} \xi^{\prime}, \eta\right)\right) \\
& +\left(\frac{p}{\sqrt{p-1}}-2\right)(\sin \alpha)\left(R_{a} \xi^{\prime}, \eta\right)+\frac{p-2}{\sqrt{p-1}}(\sin \alpha)\left(R_{s} \xi^{\prime}, \eta\right) \\
& +\frac{p-2}{\sqrt{p-1}}(\cos \alpha)\left(B_{s} \xi^{\prime}, \eta\right)
\end{aligned}
$$

where we used Lemma 2.8(iii) and (iv) in the second step. Next we estimate the terms in (28). By Lemma 2.3 we have

$$
\left|(\sin \alpha)\left(\left(B_{s} \xi^{\prime}, \xi^{\prime}\right)+\left(B_{s} \eta, \eta\right)-2\left(R_{a} \xi^{\prime}, \eta\right)\right)\right| \leq \sin (|\alpha|)(\tan \theta)\left(\left(R_{s} \xi^{\prime}, \xi^{\prime}\right)+\left(R_{s} \eta, \eta\right)\right)
$$

since $B_{a}=0$ by hypothesis. Using Lemma 2.4 and the fact that $\sin \phi=\frac{2 \sqrt{p-1}}{p}$ we deduce that

$$
\left|\left(\frac{p}{\sqrt{p-1}}-2\right)(\sin \alpha)\left(R_{a} \xi^{\prime}, \eta\right)\right| \leq\left(\frac{2}{\sin \phi}-2\right) \sin (|\alpha|)(\tan \theta)\left(\left(R_{s} \xi^{\prime}, \xi^{\prime}\right)+\left(R_{s} \eta, \eta\right)\right) .
$$

Next note that $\cot \phi=\frac{|p-2|}{2 \sqrt{p-1}}$. Therefore

$$
\left|\frac{p-2}{\sqrt{p-1}}(\sin \alpha)\left(R_{s} \xi^{\prime}, \eta\right)\right| \leq(\cot \phi) \sin (|\alpha|)\left(\left(R_{s} \xi^{\prime}, \xi^{\prime}\right)+\left(R_{s} \eta, \eta\right)\right)
$$

by Lemma 2.1, It follows from Lemma 2.2 that

$$
\left|\frac{p-2}{\sqrt{p-1}}(\cos \alpha)\left(B_{s} \xi^{\prime}, \eta\right)\right| \leq(\cot \phi)(\cos \alpha)(\tan \theta)\left(\left(R_{s} \xi^{\prime}, \xi^{\prime}\right)+\left(R_{s} \eta, \eta\right)\right) .
$$

Next (28), (29), (30), (31) and (32) together imply

$$
\begin{aligned}
P & \geq\left((1-(\tan \theta) \cot \phi) \cos \alpha-\left(\left(\frac{2}{\sin \phi}-1\right) \tan \theta+\cot \phi\right) \sin (|\alpha|)\right)\left(\left(R_{s} \xi^{\prime}, \xi^{\prime}\right)+\left(R_{s} \eta, \eta\right)\right) \\
& \geq 0
\end{aligned}
$$

where we used that fact that $\alpha \in(-\psi, \psi)$ and Lemma 2.1] in the last step. Combining (23) and (33) yields $\operatorname{Re}\left(B_{p, \alpha} u,|u|^{p-2} u \mathbb{1}_{[u \neq 0]}\right) \geq 0$ in this case.

Next we prove the second inequality for $B_{p, \alpha}$. We need the following density result.

Proposition 4.4. Let $\alpha \in(-\psi, \psi)$, where $\psi$ is given by (6) dense in $\left(D\left(B_{p, \alpha}\right) \cap W^{1, p}\left(\mathbb{R}^{d}\right),\|\cdot\|_{D\left(B_{p, \alpha}\right)}\right)$.

Proof. The claim follows from [Do16a, Proposition 4.7] (see also [Do16b, Proposition 4.23]). 
The second inequality is as follows (see [WD83, proposition 6.1] for the case when $\alpha=0$ and $B_{p}$ has real symmetric coefficients as well as [Do16a, Proposition 4.8] for the case when $\alpha=0)$.

Proposition 4.5. Suppose $B_{a}=0$. Let $p \in(1, \infty)$ be such that $\left|1-\frac{2}{p}\right|<\cos \theta$. Let $\alpha \in(-\gamma, \gamma)$, where $\gamma$ is given by (8). Then there exists an $M>0$ such that

$$
\operatorname{Re}\left(\nabla\left(B_{p, \alpha} u\right),|\nabla u|^{p-2} \nabla u \mathbb{1}_{[\nabla u \neq 0]}\right) \geq-M\|\nabla u\|_{p}^{p}
$$

for all $u \in W^{2, p}\left(\mathbb{R}^{d}\right)$ such that $\nabla\left(B_{p, \alpha} u\right) \in\left(L_{p}\left(\mathbb{R}^{d}\right)\right)^{d}$.

Proof. We consider two cases.

Case 1: Suppose $R_{a}=0$. Then it follows from Lemma 2.8 that $B_{a, \alpha}=R_{a} \sin \alpha=0$. Moreover, the condition $\alpha \in(-\psi, \psi)$ implies $\tan (\theta+|\alpha|)<\tan \phi$. Therefore [Do16a, Proposition 4.8] still applies to yield the result.

Case 2: Suppose $R_{a} \neq 0$. If $\alpha=0$, the claim follows from [Do16a, Proposition 4.8]. Therefore we may assume that $\alpha \neq 0$ for the rest of the proof. Note that $\alpha \in(-\gamma, \gamma)$ implies $(\tan \theta) \tan (|\alpha|)<\frac{1}{3}$ and $K \tan (|\alpha|)<1$, where $K$ is defined by (15). Let $\varepsilon_{0} \in(0,1 \wedge(p-1))$ be such that

$$
(\tan \theta) \tan (|\alpha|) \leq \frac{1-\varepsilon}{3-\varepsilon}
$$

and

$$
\begin{aligned}
& \left(\left(\frac{p}{\sqrt{(1-\varepsilon)(p-1-\varepsilon)}}-1\right) \tan \theta+\frac{|p-2|}{2 \sqrt{(1-\varepsilon)(p-1-\varepsilon)}}\right) \tan (|\alpha|) \\
\leq & 1-(\tan \theta) \frac{|p-2|}{2 \sqrt{(1-\varepsilon)(p-1-\varepsilon)}}
\end{aligned}
$$

for all $\varepsilon \in\left(0, \varepsilon_{0}\right)$. Let $\varepsilon \in\left(0, \varepsilon_{0}\right)$ be such that

$$
\varepsilon<\frac{\varepsilon_{0}}{32 d(1+\tan (\theta+|\alpha|))^{2} \sup _{1 \leq l \leq d}\left\|\partial_{l}^{2} C\right\|_{\infty}} .
$$

Let $u \in W^{2, p}\left(\mathbb{R}^{d}\right)$. By Lemma 4.4 we can assume without loss of generality that $u$ has a compact support. For the rest of the proof, all integrations are over the set $\left\{x \in \mathbb{R}^{d}:|(\nabla u)(x)| \neq 0\right\}$. We have

$$
\begin{aligned}
\left(\nabla\left(B_{p, \alpha} u\right),|\nabla u|^{p-2} \nabla u\right)= & -\sum_{k, l, j=1}^{d} \int\left(\partial_{j} \partial_{l}\left(e^{i \alpha} c_{k l} \partial_{k} u\right)\right)|\nabla u|^{p-2} \partial_{j} \bar{u} \\
= & -\sum_{k, l, j=1}^{d} \int e^{i \alpha}\left(\partial_{l}\left(\left(\partial_{j} c_{k l}\right)\left(\partial_{k} u\right)+c_{k l}\left(\partial_{j} \partial_{k} u\right)\right)\right)|\nabla u|^{p-2} \partial_{j} \bar{u} \\
= & -\sum_{k, l, j=1}^{d} \int e^{i \alpha}\left(\partial_{l}\left(\left(\partial_{j} c_{k l}\right)\left(\partial_{k} u\right)\right)\right)|\nabla u|^{p-2} \partial_{j} \bar{u} \\
& +\sum_{k, l, j=1}^{d} \int e^{i \alpha} c_{k l}\left(\partial_{j} \partial_{k} u\right) \partial_{l}\left(|\nabla u|^{p-2} \partial_{j} \bar{u}\right) \\
= & (\mathrm{I})+(\mathrm{II}) .
\end{aligned}
$$


We first consider the real part of (I). We have

$$
\begin{aligned}
& -\operatorname{Re} \sum_{k, l, j=1}^{d} \int e^{i \alpha}\left(\partial_{l}\left(\left(\partial_{j} c_{k l}\right)\left(\partial_{k} u\right)\right)\right)|\nabla u|^{p-2} \partial_{j} \bar{u} \\
= & -\operatorname{Re} \sum_{k, l, j=1}^{d} \int e^{i \alpha}\left(\partial_{l} \partial_{j} c_{k l}\right)\left(\partial_{k} u\right)\left(\partial_{j} \bar{u}\right)|\nabla u|^{p-2} \\
& -\operatorname{Re} \sum_{k, l, j=1}^{d} \int e^{i \alpha}\left(\partial_{j} c_{k l}\right)\left(\partial_{l} \partial_{k} u\right)\left(\partial_{j} \bar{u}\right)|\nabla u|^{p-2} \\
= & (\mathrm{Ia})+(\mathrm{Ib}) .
\end{aligned}
$$

For (Ia) we have

$$
\text { (Ia) } \geq-\frac{1}{2} \sum_{k, l, j=1}^{d}\left\|c_{k l}\right\|_{W^{2, \infty}} \int\left(\left|\partial_{k} u\right|^{2}+\left|\partial_{j} u\right|^{2}\right)|\nabla u|^{p-2} \geq-M_{1}\|\nabla u\|_{p}^{p},
$$

where $M_{1}=d^{2} \sup \left\{\left\|c_{k l}\right\|_{W^{2, \infty}}: 1 \leq k, l \leq d\right\}$. Let $U=\left(\partial_{l} \partial_{k} u\right)_{1 \leq k, l \leq d}$. For (Ib) we estimate

$$
\begin{aligned}
(\mathrm{Ib}) & =-\operatorname{Re} \sum_{j=1}^{d} \int \operatorname{tr}\left(\left(\partial_{j} C_{\alpha}\right) U\right)\left(\partial_{j} \bar{u}\right)|\nabla u|^{p-2} \\
& \geq-\sum_{j=1}^{d} \int\left(\varepsilon\left|\operatorname{tr}\left(\left(\partial_{j} C_{\alpha}\right) U\right)\right|^{2}|\nabla u|^{p-2}+\frac{1}{4 \varepsilon}\left|\partial_{j} \bar{u}\right|^{2}|\nabla u|^{p-2}\right) \\
& \geq-\varepsilon^{\prime} \int \operatorname{tr}\left(U R_{s, \alpha} \bar{U}\right)|\nabla u|^{p-2}-M_{2}\|\nabla u\|_{p}^{p} \\
& =-\varepsilon^{\prime} \int \operatorname{tr}\left(\bar{U} R_{s, \alpha} U\right)|\nabla u|^{p-2}-M_{2}\|\nabla u\|_{p}^{p},
\end{aligned}
$$

where we used Lemma 2.7 in the third step with

$$
\varepsilon^{\prime}=32 \varepsilon d(1+\tan (\theta+|\alpha|))^{2} \sup _{1 \leq l \leq d}\left\|\partial_{l}^{2} C\right\|_{\infty}
$$

and $M_{2}=\frac{1}{4 \varepsilon}$. Note that $\varepsilon^{\prime} \in\left(0, \varepsilon_{0}\right)$ by $(\underline{36})$.

Next we consider the real part of (II). Note that

$$
\begin{aligned}
\operatorname{Re} \sum_{k, l, j=1}^{d} \int e^{i \alpha} c_{k l}\left(\partial_{j} \partial_{k} u\right) \partial_{l}\left(|\nabla u|^{p-2} \partial_{j} \bar{u}\right)= & \operatorname{Re} \sum_{k, l, j=1}^{d} \int e^{i \alpha} c_{k l}\left(\partial_{j} \partial_{k} u\right)\left(\partial_{l} \partial_{j} \bar{u}\right)|\nabla u|^{p-2} \\
& +\operatorname{Re} \sum_{k, l, j=1}^{d} \int e^{i \alpha} c_{k l}\left(\partial_{j} \partial_{k} u\right)\left(\partial_{j} \bar{u}\right) \partial_{l}\left(|\nabla u|^{p-2}\right) \\
= & (\mathrm{IIa})+(\mathrm{IIb}) .
\end{aligned}
$$

In what follows we let $U \nabla \bar{u}=\xi+i \eta$, where $\xi, \eta \in \mathbb{R}^{d}$. For (IIa) we have

$$
\text { (IIa) }=\int \operatorname{tr}\left(\bar{U} \operatorname{Re}\left(C_{\alpha}\right) U\right)|\nabla u|^{p-2}=\int \operatorname{tr}\left(\bar{U} R_{s, \alpha} U\right)|\nabla u|^{p-2}+i \int \operatorname{tr}\left(\bar{U} B_{a, \alpha} U\right)|\nabla u|^{p-2} \text {. }
$$


For (IIb) we have

$$
\begin{aligned}
(\mathrm{IIb}) & =\operatorname{Re} \sum_{k, l, i, j=1}^{d} \frac{p-2}{2} \int e^{i \alpha} c_{k l}\left(\partial_{j} \partial_{k} u\right)\left(\partial_{j} \bar{u}\right)\left(\left(\partial_{l} \partial_{i} u\right)\left(\partial_{i} \bar{u}\right)+\left(\partial_{l} \partial_{i} \bar{u}\right)\left(\partial_{i} u\right)\right)|\nabla u|^{p-4} \\
& =\frac{p-2}{2} \int \operatorname{Re}\left(\left(C_{\alpha} U \nabla \bar{u}, \overline{U \nabla \bar{u}}\right)+\left(C_{\alpha} U \nabla \bar{u}, U \nabla \bar{u}\right)\right)|\nabla u|^{p-4} \\
& =(p-2) \int\left(\left(R_{\alpha} \xi, \xi\right)-\left(B_{\alpha} \eta, \xi\right)\right)|\nabla u|^{p-4} \\
& =(p-2) \int\left(\left(R_{s, \alpha} \xi, \xi\right)-\left(B_{s, \alpha} \xi, \eta\right)+\left(B_{a, \alpha} \xi, \eta\right)\right)|\nabla u|^{p-4}
\end{aligned}
$$

where $\xi, \eta \in \mathbb{R}^{d}$ and $U \nabla \bar{u}=\xi+i \eta$.

In total we obtain

$$
\begin{aligned}
\operatorname{Re}\left(\nabla\left(B_{p, \alpha} u\right),|\nabla u|^{p-2} \nabla u\right) \geq & -\left(M_{1}+M_{2}\right)\|\nabla u\|_{p}^{p}+\left(1-\varepsilon^{\prime}\right) \int \operatorname{tr}\left(U R_{s, \alpha} \bar{U}\right)|\nabla u|^{p-2} \\
& +i \int \operatorname{tr}\left(U B_{a, \alpha} \bar{U}\right)|\nabla u|^{p-2} \\
& +(p-2) \int\left(\left(R_{s, \alpha} \xi, \xi\right)-\left(B_{s, \alpha} \xi, \eta\right)+\left(B_{a, \alpha} \xi, \eta\right)\right)|\nabla u|^{p-4} \\
= & -\left(M_{1}+M_{2}\right)\|\nabla u\|_{p}^{p}+P,
\end{aligned}
$$

where

$$
\begin{aligned}
P= & \left(1-\varepsilon^{\prime}\right) \int \operatorname{tr}\left(U R_{s, \alpha} \bar{U}\right)|\nabla u|^{p-2}+i \int \operatorname{tr}\left(U B_{a, \alpha} \bar{U}\right)|\nabla u|^{p-2} \\
& +(p-2) \int\left(\left(R_{s, \alpha} \xi, \xi\right)-\left(B_{s, \alpha} \xi, \eta\right)+\left(B_{a, \alpha} \xi, \eta\right)\right)|\nabla u|^{p-4} .
\end{aligned}
$$

Next we will show that $P \geq 0$. First note that $\left(1-\varepsilon^{\prime}\right)(\cos \alpha)-\left(3-\varepsilon^{\prime}\right) \sin (|\alpha|) \tan \theta \geq 0$ due to (34). It follows that

$$
\begin{aligned}
& \left(1-\varepsilon^{\prime}\right) \operatorname{tr}\left(\bar{U} R_{s, \alpha} U\right)|\nabla u|^{2}+i \operatorname{tr}\left(\bar{U} B_{a, \alpha} U\right)|\nabla u|^{2} \\
= & \left(1-\varepsilon^{\prime}\right)(\cos \alpha) \operatorname{tr}\left(\bar{U} R_{s} U\right)|\nabla u|^{2}-\left(1-\varepsilon^{\prime}\right)(\sin \alpha) \operatorname{tr}\left(\bar{U} B_{s} U\right)|\nabla u|^{2} \\
& +i(\sin \alpha) \operatorname{tr}\left(\bar{U} R_{a} U\right)|\nabla u|^{2} \\
= & \left(\left(1-\varepsilon^{\prime}\right) \cos \alpha-\left(3-\varepsilon^{\prime}\right) \sin (|\alpha|) \tan \theta\right) \operatorname{tr}\left(\bar{U} R_{s} U\right)|\nabla u|^{2} \\
& +\left(1-\varepsilon^{\prime}\right) \sin (|\alpha|) \operatorname{tr}\left(\bar{U}\left((\tan \theta) R_{s}-\frac{\sin \alpha}{\sin (|\alpha|)} B_{s}\right) U\right)|\nabla u|^{2} \\
& +\sin (|\alpha|) \operatorname{tr}\left(\bar{U}\left(2(\tan \theta) R_{s}+i \frac{\sin \alpha}{\sin (|\alpha|)} R_{a}\right) U\right)|\nabla u|^{2} \\
\geq & \left(\left(1-\varepsilon^{\prime}\right)(\cos \alpha)-\left(3-\varepsilon^{\prime}\right) \sin (|\alpha|) \tan \theta\right)\left(R_{s} U \nabla \bar{u}, U \nabla \bar{u}\right) \\
& +\left(1-\varepsilon^{\prime}\right) \sin (|\alpha|)\left(\left((\tan \theta) R_{s}-\frac{\sin \alpha}{\sin (|\alpha|)} B_{s}\right) U \nabla \bar{u}, U \nabla \bar{u}\right)
\end{aligned}
$$




$$
\begin{aligned}
& +\sin (|\alpha|)\left(\left(2(\tan \theta) R_{s}+i \frac{\sin \alpha}{\sin (|\alpha|)} R_{a}\right) U \nabla \bar{u}, U \nabla \bar{u}\right) \\
= & \left(1-\varepsilon^{\prime}\right)(\cos \alpha)\left(R_{s} U \nabla \bar{u}, U \nabla \bar{u}\right)-\left(1-\varepsilon^{\prime}\right)(\sin \alpha)\left(B_{s} U \nabla \bar{u}, U \nabla \bar{u}\right) \\
& +i(\sin \alpha)\left(R_{a} U \nabla \bar{u}, U \nabla \bar{u}\right) \\
= & \left(1-\varepsilon^{\prime}\right)(\cos \alpha)\left(\left(R_{s} \xi, \xi\right)+\left(R_{s} \eta, \eta\right)\right)-\left(1-\varepsilon^{\prime}\right)(\sin \alpha)\left(\left(B_{s} \xi, \xi\right)+\left(B_{s} \eta, \eta\right)\right) \\
& +2(\sin \alpha)\left(R_{a} \xi, \eta\right),
\end{aligned}
$$

where we used Lemmas 2.5 and 2.6 in the third step. Hence we obtain

$$
\begin{aligned}
P \geq & \int\left(\left(1-\varepsilon^{\prime}\right)(\cos \alpha)\left(\left(R_{s} \xi, \xi\right)+\left(R_{s} \eta, \eta\right)\right)-\left(1-\varepsilon^{\prime}\right)(\sin \alpha)\left(\left(B_{s} \xi, \xi\right)+\left(B_{s} \eta, \eta\right)\right)\right. \\
& \left.+2(\sin \alpha)\left(R_{a} \xi, \eta\right)\right)|\nabla u|^{p-4} \\
& +(p-2) \int\left(\left(R_{s, \alpha} \xi, \xi\right)-\left(B_{s, \alpha} \xi, \eta\right)+\left(B_{a, \alpha} \xi, \eta\right)\right)|\nabla u|^{p-4} \\
= & \int\left((\cos \alpha)\left(\left(p-1-\varepsilon^{\prime}\right)\left(R_{s} \xi, \xi\right)+\left(1-\varepsilon^{\prime}\right)\left(R_{s} \eta, \eta\right)\right)\right. \\
& -(\sin \alpha)\left(\left(p-1-\varepsilon^{\prime}\right)\left(B_{s} \xi, \xi\right)+\left(1-\varepsilon^{\prime}\right)\left(B_{s} \eta, \eta\right)\right) \\
& \left.+p(\sin \alpha)\left(R_{a} \xi, \eta\right)-(p-2)(\sin \alpha)\left(R_{s} \xi, \eta\right)-(p-2)(\cos \alpha)\left(B_{s} \xi, \eta\right)\right)|\nabla u|^{p-4} \\
= & \int\left((\cos \alpha)\left(\left(R_{s} \xi^{\prime}, \xi^{\prime}\right)+\left(R_{s} \eta^{\prime}, \eta^{\prime}\right)\right)-(\sin \alpha)\left(\left(B_{s} \xi^{\prime}, \xi^{\prime}\right)+\left(B_{s} \eta^{\prime}, \eta^{\prime}\right)\right)\right. \\
& +\frac{p}{\sqrt{\left(1-\varepsilon^{\prime}\right)\left(p-1-\varepsilon^{\prime}\right)}}(\sin \alpha)\left(R_{a} \xi^{\prime}, \eta^{\prime}\right)-\frac{p-2}{\sqrt{\left(1-\varepsilon^{\prime}\right)\left(p-1-\varepsilon^{\prime}\right)}}(\sin \alpha)\left(R_{s} \xi^{\prime}, \eta^{\prime}\right) \\
& \left.-\frac{p-2}{\sqrt{\left(1-\varepsilon^{\prime}\right)\left(p-1-\varepsilon^{\prime}\right)}}(\cos \alpha)\left(B_{s} \xi^{\prime}, \eta^{\prime}\right)\right)|\nabla u|^{p-4},
\end{aligned}
$$

where we used Lemma 2.8](iii) and (iv) in the second step, $\xi^{\prime}=\sqrt{p-1-\varepsilon^{\prime}} \xi$ and $\eta^{\prime}=$ $\sqrt{1-\varepsilon^{\prime}} \eta$. Finally using (35) we argue in a similar manner to that used in Case 2 of the proof of Proposition 4.3 to derive $P \geq 0$. Thus it follows from (37) that

$$
\operatorname{Re}\left(\nabla\left(B_{p, \alpha} u\right),|\nabla u|^{p-2} \nabla u\right) \geq-\left(M_{1}+M_{2}\right)\|\nabla u\|_{p}^{p}
$$

as claimed.

Next we use the two inequalities obtained in Propositions 4.3 and 4.5 to show that $B_{p, \alpha}$ is $m$-accretive for all $\alpha$ in a suitable range.

Proposition 4.6. Suppose $B_{a}=0$. Let $p \in(1, \infty)$ be such that $\left|1-\frac{2}{p}\right|<\cos \theta$. Let $\alpha \in(-\gamma, \gamma)$, where $\gamma$ is given by (8). Then $B_{p, \alpha}$ is $m$-accretive.

Proof. The result follows from the arguments used in the proof of [Do16a, Proposition 4.9]. Note that [Do16a, Propositions 4.1, 4.7 and 4.8] used in the proof of [Do16a, Proposition 4.9] are now replaced by Propositions 4.3, 4.4 and 4.5 respectively. 
We now have enough preliminary results to prove Theorem 1.4,

Proof of Theorem 1.4. We consider two parts.

(i) Contractivity: Using Proposition 4.6 and [Kat80, Theorem IX.1.23] we deduce that $-B_{p}$ generates a holomorphic semigroup with angle $\psi$ given by (6) which is contractive on the sector $\Sigma_{\gamma}$, where $\gamma$ is given by (8) . Note that $B_{p}=\overline{A_{p}}$ by Proposition 4.2. Hence $S^{(p)}$ is contractive on $\Sigma_{\gamma}$.

(ii) Consistency: It suffices to show that $S^{(p)}$ is consistent with $S$. It follows from [Do16a, Propositions 1.1 and 5.1] that the $C_{0}$-semigroup generated by $-B_{2}$ is consistent with the $C_{0}$-semigroup generated by $-B_{p}$. Since $B_{2}=\overline{A_{2}}$ and $B_{p}=\overline{A_{p}}$ by Proposition 4.2 , the semigroup $S^{(p)}$ is consistent with $S$ as required.

\section{Acknowledgements}

I wish to thank Tom ter Elst for giving detailed and valuable comments.

\section{References}

[CM05] Cialdea, A. and MaZ'ya, V., Criterion for the $L^{p}$-dissipativity of second order differential operators with complex coefficients. J. Math. Pures Appl. (9) 84 (2005), 1067-1100.

[Dav89] Davies, E. B., Heat kernels and spectral theory. Cambridge Tracts in Mathematics 92. Cambridge University Press, Cambridge etc., 1989.

[Do16a] Do, T. D., Degenerate elliptic operators in $L_{p^{-}}$spaces with complex $W^{2, \infty}$ coefficients. Submitted.

[Do16b] - Degenerate elliptic second-order differential operators with bounded complexvalued coefficients. PhD thesis, The University of Auckland, New Zealand, 2016.

[DE16] Do, T. D. and Elst, A. F. M. TER, One-dimensional degenerate elliptic operators on $L_{p}$-spaces with complex coefficients. Semigroup Forum 92 (2016), $559-586$.

[Epp89] Epperson, J. B., The hypercontractive approach to exactly bounding an operator with complex Gaussian kernel. J. Funct. Anal. 87 (1989), 1-30.

[Gol85] Goldstein, J. A., Semigroups of linear operators and applications. Oxford Mathematical Monographs. The Clarendon Press, Oxford University Press, New York, 1985.

[Kat80] Kato, T., Perturbation theory for linear operators. Second edition, Grundlehren der mathematischen Wissenschaften 132. Springer-Verlag, Berlin etc., 1980.

[LP95] Liskevich, V. A. and Perelmuter, M. A., Analyticity of sub-Markovian semigroups. Proc. Amer. Math. Soc. 123 (1995), 1097-1104.

[MS08] Metafune, G. and Spina, C., An integration by parts formula in Sobolev spaces. Mediterr. J. Math. 5 (2008), 357-369. 
[Oka91] Okazawa, N., Sectorialness of second order elliptic operators in divergence form. Proc. Amer. Math. Soc. 113 (1991), 701-706.

[Ouh05] Ouhabaz, E.-M., Analysis of heat equations on domains, vol. 31 of London Mathematical Society Monographs Series. Princeton University Press, Princeton, NJ, 2005.

[Paz83] PAzy, A., Semigroups of linear operators and applications to partial differential equations. Applied mathematical sciences 44. Springer-Verlag, New York etc., 1983.

[RS75] Reed, M. and Simon, B., Methods of modern mathematical physics II. Fourier analysis, self-adjointness. Academic Press, New York etc., 1975.

[Ste70] Stein, E. M., Topics in harmonic analysis related to the Littlewood-Paley theory. Annals of Mathematics Studies 63. Princeton University Press, Princeton, 1970.

[WD83] Wong-Dzung, B., $L^{p}$-Theory of degenerate-elliptic and parabolic operators of second order. Proc. Roy. Soc. Edinburgh Sect. A 95 (1983), 95-113.

[Wei] Weissler, F. B., Two-point inequalities, the Hermite semigroup, and the GaussWeierstrass semigroup. J. Funct. Anal. 32, 102. 